\title{
Comparison of regenerative neurogenesis in response to CNS injury between adult zebrafish and mice
}

\author{
Kuan-En Chung
}

\begin{abstract}
The difference between adult zebrafish and mice in their regenerative capacity following central nervous system (CNS) injury is influenced by the permissiveness of the brain microenvironment aside from the intrinsic neurogenic potential of the cell population. In adult zebrafish, glia cells largely retain their radial characteristics and neurogenic capacity, and the zebrafish brain shows full recovery after traumatic brain injury (TBI) as well as spinal cord injury ( $\mathrm{SCl}$ ). Conversely, in mice, radial glia (RG) have largely differentiated into astrocytes. Excluding certain brain regions, following TBI, reactive astrocytes that show the potential to become neural stem cells (NSCs) in vitro remain strictly non-neurogenic in vivo due to the presence of inhibitory factors in the microenvironment. Combined with prolonged inflammation and gliosis, injury to the CNS eventually results in formation of a glial scar further impeding regeneration. However in rodents, suppression of neurogenesis may be a protection mechanism against possible detrimental side-effects of neurogenesis in the long term.
\end{abstract}

Keywords: Regeneration; Adult neurogenesis; Zebrafish; Mouse; Traumatic brain injury; Spinal cord injury

\section{Résumé}

La différence entre le poisson zèbre adulte et les souris quant à leur capacité de régénération après une lésion du système nerveux central (SNC) est influencée par la permissivité du microenvironnement du cerveau, mis à part le potentiel neurogène intrinsèque de la population cellulaire. Chez le poisson zèbre adulte, les cellules gliales conservent en grande partie leurs caractéristiques radiales et leur capacité neurogène. De plus, le cerveau du poisson zèbre démontre un rétablissement complet après une lésion cérébrale traumatique (LCT) et une lésion de la moelle épinière (LME). Inversement, chez les souris, les cellules gliales se différencient en astrocytes. En excluant certaines régions du cerveau, après une LCT, les astrocytes réactifs qui ont le potentiel de devenir des cellules souches neurales (CSN) in vitro demeurent strictement non-neurogènes in vivo, en raison de facteurs inhibiteurs présents dans le microenvironnement. Combinée avec une inflammation prolongée et une gliose, une lésion du SNC aboutit finalement à la formation d'une cicatrice gliale qui entrave encore la régénération. Cependant, chez les rongeurs, la suppression de la neurogenèse peut constituer un mécanisme de protection contre les effets éventuels de la neurogenèse à long terme.

Mots Clés: Régénération; Neurogenèse adulte; Poisson zèbre; Souris; Lésion cérébrale traumatique; Lésion de la moelle épinière

\section{Introduction}

The adult zebrafish has an excellent ability to regenerate its CNS following TBI. TBI is characterized by physical injury to the brain that is not the result of a natural disease, in contrast to non-TBI such as neural degeneration due to stroke. Whereas mammals lack the capacity to do so save for a few areas such as the hippocampus (1).
Since regenerative capacities differ even across teleost and mammalian species, this review focuses on the comparison between zebrafish and mice specifically. It investigates the conserved molecular mechanisms regarding neurogenesis and neural stem cell (NSC) response following injury to the CNS, and reflects on the reasons of these differences from an evolutionary standpoint.

Correspondence: kchun091@uottawa.ca

Department of Biology, University of Ottawa, 30 Marie Curie, K1N 6N5,

Ottawa, Canada 


\section{Regeneration in adult zebrafish CNS}

Under physiological conditions, there is widespread neurogenesis in the zebrafish brain (2). While glial fibrillary acidic protein-positive $(\mathrm{GFAP}+) \mathrm{RG}$ cells that reside in the ventricular zone (VZ) are a major NSC component (3), many other neuronal progenitors do not show GFAP or radial characteristics. For instance, neuroepithelial (NE) cells dominate the stem cell population for cerebellar regeneration (4). Even neurogenesis pathways show considerable heterogeneity: two distinct modes of neurogenesis, dorsal and ventral have been found in the zebrafish respectively in the dorsal and ventral forebrain. The dorsal stem cell niche is dominated by RG, while the ventral stem cell niche mainly has an NE population and shows more dependence on Fgf signalling (5). While previous research has suggested similarities between certain ventral stem cells of the zebrafish and the progenitors of the mammalian SVZ (6), the role of Fgf in the latter is less defined.

The well-known outcome in adult zebrafish following TBI is reactive neurogenesis and complete regeneration, yet only the response in the dorsal telencephalic NSC niche has been characterized to date. In response to injury, telencephalic RG cells proliferate and generate neuroblasts that migrate to the lesion site. Major responding RG cells express the her 4.1 gene, a target of Notch signaling, and they are thought to act directly as neuronal progenitors without undergoing dedifferentiation to a less specialized cellular state (7). The entire process is initiated by an acute inflammatory response and reactive gliosis that subsides within a few days: up-regulation of the M2 macrophage secretes antiinflammatory cytokines, while microglia clears up toxic debris. Moreover, the inflammation event itself directly activates radial glial cells through cysteinyl leukotriene signalling (8). Subsequent neurogenesis quickly replaces the damaged neurons.

A successful regeneration consists of not only the activation of stem cells, but also incorporation into the existing circuit. While the details of successful neuronal integration into the adult zebrafish circuitry have not been examined to date, several factors have been identified. They could be proliferation factors that recruit NSCs, or permissive factors that allow the development of NSCs into long lasting neurons. Wnt and Notch signalling are known thus far to be involved in radial glia proliferation and neurogenesis following TBI: Wnt induces proliferation, while the RG-repressing Notch indirectly allows proliferation through its own decrease upon injury (2). As well, the cxcr5 gene expression in RG is shown to permit its differentiation into neurons $(9)$.

Differences also lie in the migration of newborn neurons to the injury site. For adult spinal cord injury in mammals, the failure of axons to navigate to the injury site may underlie the absence of neuronal replacement (10). Whereas for adult zebrafish, Fgf signalling induces radial glia to form a "glial bridge" that allows for newly formed axons to migrate over to the injury site. RG progenitors thus act as a navigation guide for their own derived neurons in the injury response (11). It is noteworthy that mice do show Fgf signalling during embryonic development, but it is down regulated after birth. When mouse astrocytes are treated with Fgf, they show the same potential to form a glial bridge (11). In addition of Notch1, connective tissue growth factor a (ctgfa) expression in and around radial glia helps the bridging and migration process following spinal cord injury in adult zebrafish (12). Though there is no evidence of ctgf playing a similar role in adult mice, it does promote gliogenesis in embryos (13) suggesting a conserved mechanism.

\section{Regeneration in adult mouse mammalian CNS}

One crucial difference between the zebrafish and the mice in their regenerative capacity is the absence of neurogenic radial glial cells in adult mice. Mammals have radial glia that originate from NE during embryonic CNS development, but the radial glia NSCs subsequently differentiate into intermediate progenitor cells (IPCs) which then give rise to astrocytes and oligodendrocytes (14). Astrocytes are developed from GFAP+ cells that lose their radial characteristic and become more differentiated (15). They still share the most morphological similarities with radial glia out of all mammalian glia types, but specialize in maintaining homeostasis at the cost of regenerative function. However, introduction of brain-derived neurotropic factor (BDNF) has shown to induce adult neurogenesis in areas including the striatum and septum of the lateral and third ventricles, as well as the thalamus and hypothalamus that were not expected to show such activity (16), and astrocytes have been shown to generate neurons upon stimulation in vitro, though fail to do so in vivo (17). This restriction may therefore be in part contributed by the microenvironment of the mammalian brain.

Regenerative neurogenesis in mice does occur in the dorsal subgranular zone (SGZ) of the hippocampus and the ventral subventricular zone (SVZ) of the lateral ventricles. Unlike the rest of the brain, astrocytes in these areas actively regulate differentiation and integration of new neurons (18). Successful neurogenesis here is thought to be regulated by a variety of signalling factor in common with the zebrafish such as Wnt, Shh, Notch, BMP, neurotrophin and neurotransmitters (19). The NSC population comprises of Type B astrocytes in the SVZ and Type 1 (also known as radial glia-like) cells in the SGZ before differentiating into neuroblasts (19). 
Interestingly, the extent of NSC proliferation and neuron maturation is somewhat dependent on the extent of TBI (20). But in contrast to zebrafish, even after integration of the new neurons into the brain circuit, synapses remained immature in morphology (21) or shows altered morphology compared to surrounding neurons with unclear functional impacts in the long run (22).

Unlike the zebrafish, the response of adult mice following TBI in brain regions outside of the SVZ and SGZ does not, save for the olfactory bulb, result in neuronal regeneration. Instead, intense inflammation and glial scarring are observed in complete contrast to the zebrafish. Although reactive astrocytes - astrocytes activated specifically following TBI-have the potential to be multipotent NSCs in vitro, the non-permissive environment in vivo prevents them from realizing this ability (7). What actually happens is that, in response to injury, NSCs proliferate and differentiate into neural progenitor cells (NPCs) that migrate from the SVZ (and the SVZ only) to the lesion site, just like injuries in the SVZ/SGZ. The proliferation factors associated include the rapamycin signaling pathway activation (23), Notch 1 (24). Chemokines expressed by macrophages and microglia attract NPCs to the injury site (25). The same signalling is not found in zebrafish. However, newborn SVZ neurons do not persist long nor mature where they need to be, that is, at the lesion site. This is thought to be due to extrinsic factors at the lesion site outside of the stem cell niche limiting SVZ differentiation. Surviving SVZ cells all end up becoming glial cells, not neurons that are needed to replace cell loss $(26,27)$.

There are several observable differences between the zebrafish and the mouse response to CNS injury. Unlike in zebrafish, the mammalian inflammatory response alone initiates gliosis, secondary tissue damage and lesion cavity expansion (28). Immediately following TBI, reactive astrocytes are mass recruited around the lesion site and promote extracellular matrix (ECM) protein deposition in forming the glial scar. However, astrogliosis at this stage serves to preserve tissue and repair the blood-brain barrier around the lesion site (29). Furthermore, recent research has demonstrated that astrocyte scar formation may actually support neuronal regrowth but only under artificial supply of axon-specific growth factors (30). While the early inflammation response activates both the pro-inflammatory M1 and the anti-inflammatory M2 immune cells, only the M1 response remains over time while the M2 deteriorates (31). Circulating macrophage and microglia then contribute to secondary tissue damage: Microglia releases pro-inflammatory neurotoxic and cytotoxic molecules such as cytokines and nitric oxide. Following the acute phase, neuroinflammation persists and spreads to regions surrounding the lesion site. The characteristic glial scar is the result of accumulation of astrocytes, extracellular protein deposition, persistence of leukocytes and inhibition of axonal regeneration around the lesion site, all leading to permanent scarring. There appears to be no benefit associated with the chronic response: it simply results in progressive brain deterioration, immune cell infiltration, and pro-inflammatory cytokines that suppress neurogenesis (32). Studies that aim to control the chronic response has shown to limit degeneration and improve functional recovery, but improvements are limited to the female mice (33). Interestingly, where inflammation can be avoided, axons have demonstrated an ability to extend even amidst degenerating white matter until they reach into the lesion site. This indicates that rather than universally hostile to regeneration, the reactive glial matrix at the lesion site itself appears to be the major barrier to neuronal growth (34). However, even in a dystrophic growth cone, the neuronal endings remain dynamic in their motility (35).

What stops growth cones at the site of the scar? Deposition of ECM debris during scarring mechanically blocks the axons, but there are also signals that actively inhibit them, in accordance with the assumption that it is the microenvironment around the lesion site that prevents neurogenesis. Several CNS myelin-derived proteins including the Nogo family, MAG, OMgp all work through the Nogo-66 receptor (NgR) to suppress neurogenesis (36). However, targeting Nogo does not increase neurogenesis despite improving functional recovery (37). In addition, reactive astrocytes release chondroitin sulphate proteoglycans (CSPGs) which inhibit axon growth. Even the function of these signalling factors differs between the mice and the zebrafish. In zebrafish, Nogo is actually growth permissive due to lacking a receptor domain necessary for growth inhibition (38), while CSPGs can guide optic nerve regeneration (7). And whereas cxcr5 is responsible for radial glia differentiation into neurons in specific niches, the same gene in mice enhances SGZ cell proliferation but suppresses NPC population (39). Moreover, rho kinase, a molecule that inhibits neuron survival in mice (40) does not seem to work the same way in zebrafish besides aiding in neuronal development. Another inhibitory factor in mice is Olig2 which suppresses Pax6, a gene able to convert astrocytes into neurons in vitro. Suppression of Olig2 can lead to neurogenesis in vivo (17).

\section{Evolutionary considerations}

Similarities between SGZ/SVZ in mouse and neurogenic niches in the lateral areas of the dorsal/ventral telencephalon in zebrafish have been proposed $(5,6,41)$ regarding their common function (42), neuroanatomy (43) or neuronal migration $(44,45)$, but not investigated in detail. A question is whether the vertebrate brain is by default permissive for neuronal regeneration while mammals have lost this capacity or somehow inhibited this 
function; or it is by default inhibitory, but zebrafish have found a way to overcome this mechanism. Given the functional similarities between astrocytes and RG, and considering mechanisms retained in the mouse embryo but not the adult, the former scenario could be more likely. In this case, then, the course of mammalian evolution may eventually have selected against regeneration except for certain brain regions such as the hippocampus.

Besides the obvious advantage that adult neurogenesis confers to the organism in recovery from injuries, mice studies have also demonstrated the adaptive role of a neurogenic hippocampus in facilitating learning by optimizing new information storage $(46)$. Neural networks in learning encounter the problem known as catastrophic interference, which is a dilemma between plasticity and stability of existing circuits. Interference of the existing circuitry may lead to loss of both old and new information. Neurogenesis in the hippocampus may have served as a strategy to resolve catastrophic interference by providing new neurons that store novel information without interfering with the existing circuit (47). On the other hand, neurogenesis may also aid learning by modifying the existing circuit, thereby clearing less relevant older memories to prevent them from interfering with new ones (48). Hippocampal neurogenesis is thus positively correlated with forgetting/new learning and negatively correlated with retention, and as such, mammals in adulthood generally decrease neurogenesis levels to resist changes to memory formation (49). For zebrafish, however, where survival largely depends on detecting chemical and visual cues in the aqueous environment rather than complex learning, neural plasticity is more exemplified in chemical and visual processing centers of the brain (50). In both scenarios, the adult neurogenesis response enhances the processing of novel information in an enriched environment. However, this may not always be the case for zebrafish; studies have shown that deprivation and novelty impact plasticity not as opposing factors, but both contribute in a stage-specific manner that is relative to the environmental context throughout time (51).

Even ignoring circuit interference, disadvantages of adult regenerative neurogenesis also exist. For mammals, recent discovery shows that the neurogenic burst following TBI actually depletes finite NPC storage in the hippocampus, leading to future decline in even basal rates of neurogenesis, but also increases susceptibility to chemical seizures (52). Perhaps, regular neurogenesis in zebrafish may render it immune to this disadvantage, yet robust neurogenic activity also has its downsides, namely brain tumour if improperly regulated. While tumour incidences are among one of the lowest in the brain (53), cancerous cells rise predominantly around the SVZ where neurogenesis is prominent. Less research has been done on tumour development in zebrafish focusing on its relationship to adult neurogenesis, however, overexpression of the certain genes that lead to tumours in mammals such as $K R A S$ do not necessarily induce the same in zebrafish, suggesting different regulators of brain tumours across species (54).

Even the glial scar has proved to be of more value than it appears. Glial scarring in the early stage following TBI is integral to preserving tissue functionality around the lesion site (29). It may be that scarring to stop bleeding was more important in mammals' survival. For its part, zebrafish bypasses scar formation altogether by rapid replacement through neurogenesis following cell death (55). The same occurs in developing mammals until the radial glia are substituted by astrocytes, which may be a trade-off for specialized homeostasis at the cost of ready regeneration. Another possibility is that complete neuronal regeneration in adults may simply deplete too much energy for mammals. Given the complexity of the mammalian brain, rebuilding synaptic connections without disrupting the original circuit may involve a more complicated process that cost too much in energetics to be fully functional; whereas if partial repair ends up being more detrimental, discarding neurogenesis altogether may have been preferable. However, the consequences of temporary induction of neurogenesis following TBI are not yet clear. It is therefore suggested that more attention be drawn to potential adverse sideeffects of overriding the inhibition that the mammalian brain has imposed on its own regenerative response.

\section{Conclusion}

The regenerative capacity difference between adult zebrafish and mice is in part contributed by inhibitory microenvironmental factors in the mammalian CNS which might limit an otherwise potentially neurogenic glia population repopulate the injured region. This inhibition may have evolved as an adaptive response to detrimental effects associated with neurogenesis. Given that the long term success of artificially inducing NSC proliferation remains ambiguous, future research that follows the survival and integration of newborn neurons as well as the fate of NSC populations post CNS injury could better characterize the possible roles that adult neurogenesis might play in mammals.

\section{Competing interests}

The author declare that she has no competing interests.

\section{References}

1. C. Kizil, J. Kaslin, V. Kroehne, M. Brand, Dev Neurobiol 72, 429 (2012).

2. A. Alunni, L. Bally-Cuif, Development 143, 741 (2016).

3. K. Johnson, et al., Glia 64, 1170 (2016).

4. J. Kaslin, V. Kroehne, J. Ganz, S. Hans, M. Brand, Development 144, 3388 (2017) 
5. J. Ganz, J. Kaslin, S. Hochmann, D. Freudenreich, M. Brand, Glia $\mathbf{5 8}$ 1345 (2010).

6. B. Adolf, et al., Dev. Biol. 295, 278 (2006).

7. V. Kroehne, D. Freudenreich, S. Hans, J. Kaslin, M. Brand, Development 138, 4831 (2011).

8. N. Kyritsis, et al., Science 338, 1353 (2012).

9. C. Kizil, et al., Neural Dev 7, 27 (2012).

10. M. Kerschensteiner, M. E. Schwab, J. W. Lichtman, T. Misgeld, Nat. Med. 11, 572 (2005).

11. Y. Goldshmit, et al., J. Neurosci. 32, 7477 (2012)

12. M. H. Mokalled, et al., Science 354, 630 (2016)

13. F. A. Mendes, et al., PLoS ONE 10, e0133689 (2015).

14. A. Kriegstein, A. Alvarez-Buylla, Annu. Rev. Neurosci. 32, 149 (2009).

15. N. Kishimoto, K. Shimizu, K. Sawamoto, Disease Models \& Mechanisms 5, 200 (2012).

16. V. Pencea, K. D. Bingaman, S. J. Wiegand, M. B. Luskin, J. Neurosci. 21, 6706 (2001)

17. A. Buffo, et al., Proc. Natl. Acad. Sci. U.S.A. 102, 18183 (2005).

18. H. Song, C. F. Stevens, F. H. Gage, Nature 417, 39 (2002).

19. A. M. Bond, G. L. Ming, H. Song, Cell Stem Cell 17, 385 (2015).

20. X. Wang, X. Gao, S. Michalski, S. Zhao, J. Chen, J. Neurotrauma 33 , 721 (2016)

21. H. Nakatomi, et al., Cell 110, 429 (2002).

22. L. E. Villasana, G. L. Westbrook, E. Schnell, Exp. Neurol. 261, 156 (2014).

23. X. Wang, P. Seekaew, X. Gao, J. Chen, eNeuro 3 (2016).

24. X. Wang, X. Mao, L. Xie, D. A. Greenberg, K. Jin, J. Cereb. Blood Flow Metab. 29, 1644 (2009)

25. R. J. Gordon, A. L. McGregor, B. Connor, Mol. Cell. Neurosci. 41, 219 (2009).

26. K. Yoshiya, et al., J. Neurotrauma 20, 1147 (2003).

27. E. H. Chang, et al., Front Neurosci 10, 332 (2016).

28. M. T. Fitch, C. Doller, C. K. Combs, G. E. Landreth, J. Silver, J. Neurosci. 19, 8182 (1999).

29. J. R. Faulkner, et al., J. Neurosci. 24, 2143 (2004).

30. M. A. Anderson, et al., Nature 532, 195 (2016).

31. A. Kumar, D. M. Alvarez-Croda, B. A. Stoica, A. I. Faden, D. J. Loane, J. Neurotrauma 33, 1732 (2016).

32. S. A. Acosta, et al., PLoS ONE 8, e53376 (2013).

33. Ert .

34. S. J. Davies, D. R. Goucher, C. Doller, J. Silver, J. Neurosci. 19, 5810 (1999).

35. V. J. Tom, M. P. Steinmetz, J. H. Miller, C. M. Doller, J. Silver, J. Neurosci. 24, 6531 (2004).

36. K. C. Wang, et al., Nature 417, 941 (2002).

37. D. J. Shepherd, S. Y. Tsai, T. E. O'Brien, R. G. Farrer, G. L. Kartje, Front Neurosci 10, 467 (2016).

38. H. Abdesselem, A. Shypitsyna, G. P. Solis, V. Bodrikov, C. A. Stuermer, J. Neurosci. 29, 15489 (2009)

39. M. J. Stuart, F. Corrigan, B. T. Baune, J Neuroinflammation 11, 31 (2014).

40. N. Bye, K. J. Christie, A. Turbic, H. S. Basrai, A. M. Turnley, Exp. Neurol. 279, 86 (2016).

41. J. Kaslin, J. Ganz, M. Brand, Philos. Trans. R. Soc. Lond., B, Biol. Sci. 363, 101 (2008).

42. C. Broglio, et al., Brain Res. Bull. 66, 277 (2005).

43. C. Salas, C. Broglio, F. Rodriguez, Brain Behav. Evol. 62, 72 (2003).

44. C. A. Byrd, P. C. Brunjes, Ann. N. Y. Acad. Sci. 855, 274 (1998).

45. H. Grandel, J. Kaslin, J. Ganz, I. Wenzel, M. Brand, Dev. Biol. 295, 263 (2006).

46. G. Alexander, R. Ingo, K. Gerd, Hippocampus 26, 261 (2016).

47. W. Laurenz, R. M. J., K. Gerd, Hippocampus 16, 329 (2006).

48. P. W. Frankland, S. A. Josselyn, Neuropsychopharmacology 41, 382 (2016).

49. K. G. Akers, et al., Science 344, 598 (2014).

50. B. W. Lindsey, S. Di Donato, J. Kaslin, V. Tropepe, Eur. J. Neurosci. 40, 3591 (2014).

51. B. W. Lindsey, V. Tropepe, Dev Neurobiol 74, 1053 (2014).

52. E. J. Neuberger, B. Swietek, L. Corrubia, A. Prasanna, V. Santhakumar, Stem Cell Reports 9, 972 (2017).
53. B. A. Kohler, et al., J. Natl. Cancer Inst. 103, 714 (2011).

54. S. Konefal, M. Elliot, B. Crespi, Front Neuroanat 7, 21 (2013).

55. E. V. Baumgart, J. S. Barbosa, L. Bally-Cuif, M. Gotz, J. Ninkovic, Glia 60, 343 (2012). 